\title{
ANTICROSSINGS IN THE ACETYLENE MOLECULE FOURIER TRANSFORM ANALYSIS: MEAN COUPLING, LEVEL DENSITY
}

\author{
P. DUPRE
}

Service National des Champs Intenses, C.N.R.S., BP. 166X, F-38042 Grenoble cedex 9, France

\begin{abstract}
By using a home made dye laser we excite, in the U.V. range, the acetylene molecule $\left(\mathrm{C}_{2} \mathrm{H}_{2}\right)$ in gas phase. Four rotationless vibrational levels have been selected to obtain anticrossing spectra up to 8 tesla. To statistically analyze this data we have developped a method based on a Fourier Transform (TF) technique and on a fitting of the resulting curves. This first analysis gives the probability law of the singlet-triplet coupling for the different excited levels. A second analysis allows to extract from the first analysis of the data the vibrational level densities $\left(\rho_{v i b}\right)$, the products $\rho_{v i b} \cdot \bar{V}_{s t}$ and the mean couplings $\left(\bar{V}_{s t}\right)$. Finally we will present the variation of the product $\rho_{v i b} \cdot \bar{V}_{s t}$ as an increasing exponential fonction of the excitation energy.
\end{abstract}

\section{The laser}

We use an home made dye laser $[1,2]$ to excite the acetylene molecule. The dye laser (an oscillator cavity and two amplifier stages) is pumped by a frequency tripled and Q-switched Nd:YAG laser (QUANTEL : output energy $\sim 40 \mathrm{~mJ}$ per pulse at $355 \mathrm{~nm}$ ). We frequency double the blue dye laser radiation $\left(440-500 \mathrm{~nm}\right.$ ) with a $\beta-\mathrm{BaB}_{2} \mathrm{O}_{4}$ crystal (Fujian Institute of Research, China) giving a U.V. beam whose typical energy is $500 \mu J$ with pulse of $6 n s$ duration. The spectral bandwidth is $\sim 4 \mathrm{GHz}(\mathrm{fwhm})$ and the divergence is less than $0.4 \mathrm{mrad}$. We use a tellurium oven to calibrate the blue wavelength. The wavelength scanning is obtained by a stepping motor (Micro-Controle) rotating the back cavity mirror.

\section{The experimental Setup}

The good quality of the U.V. laser beam permits us to send the beam inside a Bitter magnet [3], at a distance of 6 meters from the laser device by using only one focusing lens ( $\sigma$ excitation). Inside the magnet, additional coils are used to give a satisfactory homogeneity of the magnetic field $\therefore$ spatial homogeneity of $0.1 \mathrm{mT}$ per $\mathrm{cm}^{-1}$ and a temporal stability of $2.510^{-5}$. The fluorscent light $(\pi)$ is collected from the cell inside the magnet by a couple of lenses and a couple of serial light pipes. A photomultiplier converts the fluorescence photons in an electrical signal which is digitized by a transient recorder and stored on a personal computer (PC). This PC is also used to trim the magnetic field. We use a NMR probe to calibrate precisely the spectra on the magnetic field every 0.1 tesla during the scans of 8 tesla (one hour for a 65536 data point record). Each record can be added to the others so as to increase the signal/noise ratio (see figure 1).

\section{Principles of the experiment and of the analysis}

In order to decrease the complexity of the spectra we optically select levels without rotation $(N=0, K=0)[3]$ of 4 vibrational levels including the $\nu_{3}$ vibrational mode : $\left(n_{3}=0-3\right.$, 


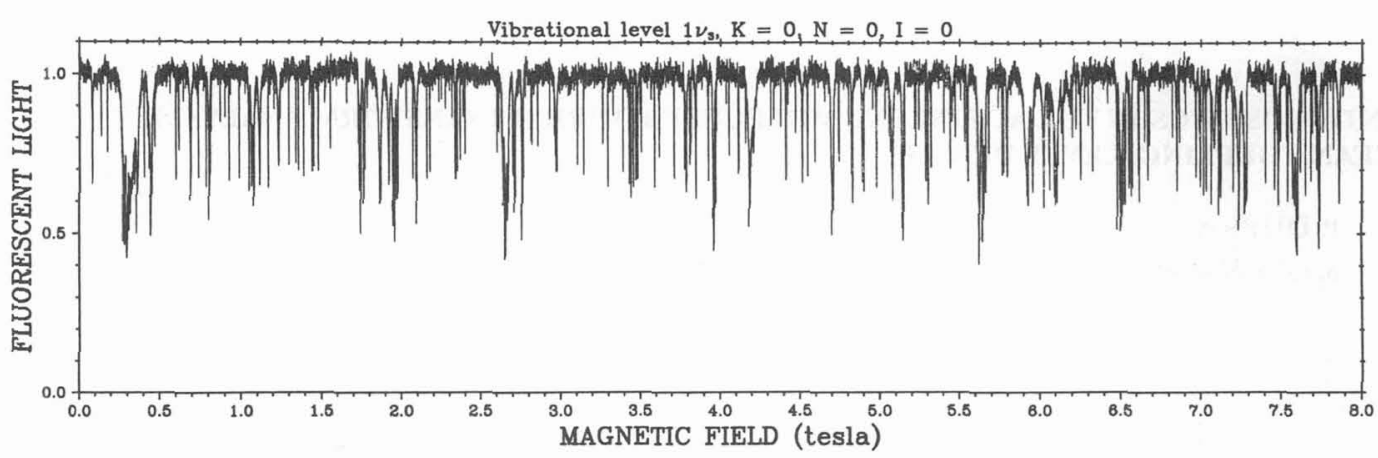

Figure 1: Anticrossing spectrum of the rotationless $1 \nu_{3}$ vibrational level, $\lambda=231.12 \mathrm{~nm}$ (65536 data points, 8 averaged spectra).

$\left.\nu_{3} \simeq 1000 \mathrm{~cm}^{-1}\right)$; electronic transition : $S_{0}\left({ }^{1} \Sigma_{g}^{+}\right) \leftrightarrow S_{1}\left({ }^{1} A_{u}\right)$. The magnetic field $(B)$ is used to modify the energy of the triplet levels by Zeeman effect $\left(E_{t}=g \mu_{B} m_{S}\left(B-B_{0}\right)\right.$, where $g$ is the gyromagnetic factor and $\mu_{B}$ is the Bohr magneton) and where the energy of the excited singlet level is unchanged. If the singlet and triplet levels are coupled and when they have nearly the same energy, one part of the excitation energy is transferred from the singlet level (optically active) to the triplet level (dark level) thereby decreasing the fluorescence light from the excited singlet level. This is called an anticrossing (AC). The theoretical shape of one AC is lorentzian and the center of the line $\left(B_{0}\right)$ is where we had a level crossing if we do not have coupling (avoided crossing). The linewidth of one $\mathrm{AC}$ is given by the value of the matrix element $(V)$ in a two level model.

By supposing that an AC spectra can be modelled by a sum of individual AC we have :

$$
I_{s p} \propto 1-\sum_{i} \frac{\alpha_{i}}{1+\left(\frac{B-B_{0_{i}}}{\Delta B_{i}}\right)^{2}}
$$

with : $\Delta B_{i}=\frac{2 V_{i}}{g \mu_{B}}$ and where the parameters $\alpha_{i}, B_{0_{i}}$ and $V_{i}$ are independant and stochastic variables with the respective probability laws : $p\left(\alpha_{i}\right), p\left(\Delta B_{0 i}\right)$ and $p\left(V_{i}\right)$. Taken the squared FT of formula (1) we have :

$$
|\mathcal{J}(\tau)|^{2} \propto\left(1-2 \sum_{i} \alpha_{i} \pi \Delta B_{i}\right) \delta_{0}(\tau)+\pi^{2} \sum_{i} \alpha_{i}^{2}\left(\Delta B_{i}\right)^{2} e^{-4 \pi \Delta B_{i}|\tau|}+\mathcal{N}
$$

by supposing that the additional term $\mathcal{N}$ is only due to stochastic noise independant of $\tau$, we assume that we do not have position correlation which could give an additional structure such a correlation hole [4]. If we neglect the terms $\delta_{0}(\tau)$ in the relation (2) we obtain a sum of decreasing exponentials.

\section{Discussion}

In figure (2) we have drawn (points) the squared FT of the figure (1) in log- log scale. As we can immediatly see, between 10 and $2000 T^{-1}$ the points are distributed along a straigth line. This behaviour has been observed for the AC FT spectra of the 4 vibrational levels. It means that the fonction $|\mathcal{J}(\tau)|^{2}$ follows a power law $\left(|\mathcal{J}(\tau)|^{2}=\mathcal{J}_{0}^{2} \cdot \tau^{\gamma}\right)$ at least over the $\tau$ domain concerned. The flat slope that we observe for the low values of $\tau\left(\tau<10 T^{-1}\right)$ is due to the fact that we do not have large $\mathrm{AC}$; the shape of the curve seems to indicate a cut phenomenon for the large couplings $\left(\Delta B_{m a x}\right)$, the value of $\Delta B_{m a x}$ increasing as the excitation energy increases. 


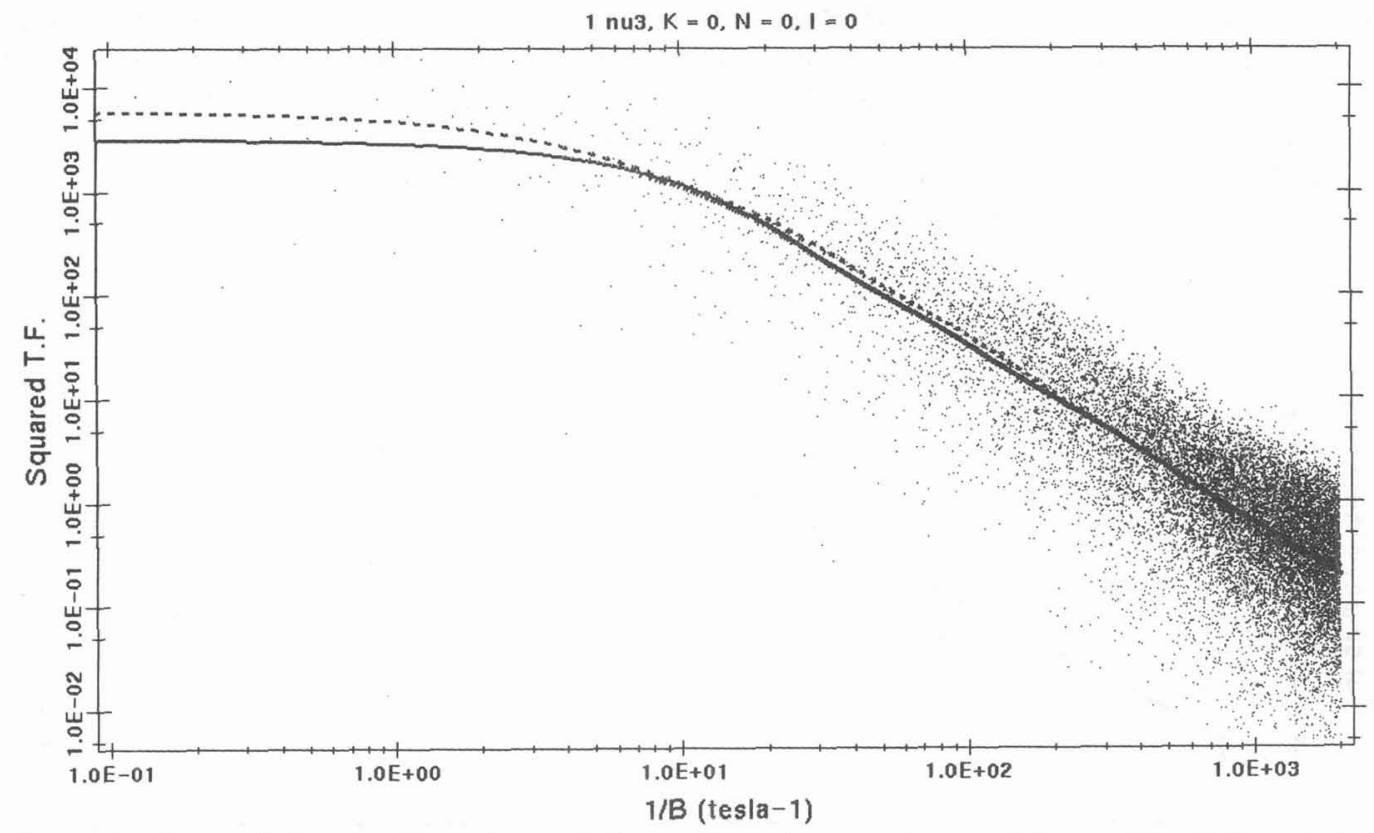

Figure 2: Squared Fourier Transform of the anticrossing spectrum of figure (1). Full and dotted lines are the results of 2 different parameter fits based on the relation (2).

If we now introduce the probability law of the coupling $p(\Delta B)$ (limited to the range $\left[\Delta B_{\text {min }}\right.$, $\Delta B_{\max }$ ]) in the formula (2) so as to work with a continue integral rather than with a discrete sum we obtain (supposing $\alpha$ constant) :

$$
|\mathcal{J}(\tau)|^{2} \propto \pi^{2} \int_{\Delta B_{\min }}^{\Delta B_{\max }} \alpha^{2}(\Delta B)^{2} p(\Delta B) e^{-4 \pi \Delta B \tau} d(\Delta B)
$$

By taken $\Delta B_{\min }=0$ and $\Delta B_{\max }=\infty$, and assuming that the probability law is a power law $\left(p(\Delta B)=(\Delta B)^{\beta}\right)$ the integral can be calculated (gamma function) :

$$
|\mathcal{J}(\tau)|^{2} \propto\left(\frac{1}{4 \pi \tau}\right)^{-\gamma}
$$

This result is in agreement with our experimental data (at least on the already mentionned $\tau$ ) and we can determine the power $(\beta=\gamma-3)$ corresponding to such a power law. From the squared FT we fit (careful attention is taken to the weighting) the values of $\gamma$ and of $\Delta B_{\max }$. The value of $\Delta B_{\min }$ will be assumed a constant and in fact this value is experimentally fixed by the magnetic field inhomogeneities ( 3 Gauss).

The analysis method that we present here is powerful and has been used to determine first the coefficient $\beta$ of the probability power law for four rotationless vibrational levels in the acetylene molecule. The obtained values are roughly similar (see table I). Supposing now that the amplitude of the AC $(\alpha)$ is fixed and known we may deduce the value of the AC densities $\left(\rho_{a c}\right)$ and supposing that, in a rough approximation, the $\mathrm{AC}$ are only singlet-triplet $\mathrm{AC}$ (no induced AC) we deduce the product $\left(\rho_{v i b} \cdot \bar{V}_{s t}\right)$, the mean singlet-triplet coupling $\left(\bar{V}_{s t}\right)$ and the triplet vibrational level density $\left(\rho_{v i b}\right)$ : see table (I); selection rules (see ref. [5]) and $g=2$ are assumed. We report in figure (3) 
the product $\left(\rho_{v i b} \cdot \bar{V}_{s t}\right)$, the basic factor which tracts the intra- molecular energy redistribution, on a semi-logarithmic scale as a function of the excitation energy. We observe roughly exponential evolution $\left(\rho_{v i b} \cdot \bar{V}_{s t}=\rho_{0} \cdot \bar{V}_{0} e^{E / E_{0}}, \rho_{0} \cdot \bar{V}_{0}=1.24410^{-38}, E_{0}=520.3 \mathrm{~cm}^{-1}\right)$. The point representing the result for the $3 \nu_{3}$ mode is not aligned with the others because the value of the product $\rho_{a c} \cdot \bar{V}$ is near 1 , corresponding to a non negligible effet of the AC overlap (not analysed here).

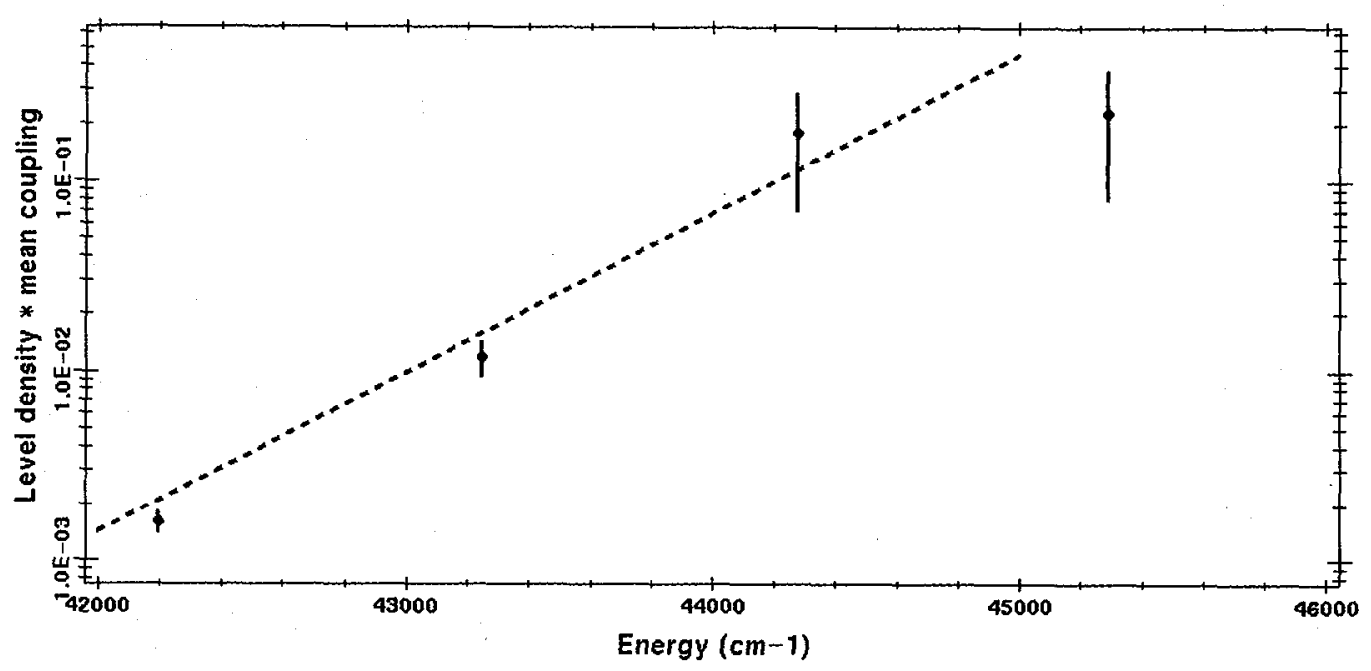

Figure 3: The evolution of the product $\rho_{v i b} \cdot \bar{V}_{s t}$ as a function of the excitation energy for 4 rotationless vibrational levels $\left(n_{3}=0-3\right)$ in a semi-logarithmic scale.

The present method has been used to analyze 18 rotational levels between $42137 \mathrm{~cm}^{-1}$ and $46290 \mathrm{~cm}^{-1}[6]$.

\begin{tabular}{|c|c|c|c|c|}
\hline & $\beta$ & $\rho_{\text {vib }}\left(\right.$ per $\left.\mathrm{cm}^{-1}\right)$ & $\rho_{v i b} \cdot \bar{V}_{s t}$ & $\bar{V}_{s t}(M H z)$ \\
\hline $0 \nu_{3}$ & $-1.31(13)$ & $0.96(12)$ & $1.6(2) \times 10^{-3}$ & $25(6)$ \\
\hline $1 \nu_{3}$ & $-1.36(9)$ & $3.74(4)$ & $11.7(2.5) \times 10^{-3}$ & $46(17)$ \\
\hline $2 \nu_{3}$ & $-1.36(4)$ & $5.3(8)$ & $0.18(11)$ & $510(390)$ \\
\hline $3 \nu_{3}$ & $-1.38(5)$ & $7.1(9)$ & $0.23(15)$ & $390(530)$ \\
\hline
\end{tabular}

Table I : Main results on the rotationless levels (from squared TF)

[1] P. Dupré, Journal de Physique, 48, C7-581 (1987)

[2] P. Dupré, Aplied Optics, 266, 860 (1987).

[3] P. Dupré, R. Jost, M. Lombardi, P.G. Green, E. Abramson and R.W. Field, Chemical Physics, 152, 293 (1991).

[4] L. Leviandier, M. Lombardi, R. Jost and J.P. Pique, Physical Review Letters, 56, 2449 (1986).

[5] P. Dupré, R. Jost and M. Lombardi, Chemical Physics, 91, 355 (1984).

[6] P. Dupré unpublished results. 